Espacio, Tiempo y Forma, Serie VI, Geografia, t. 15, 2002, págs. 29-50

\title{
Calidad e identificación en el desarrollo de la horticultura en el País Vasco
}

\author{
Juan Cruz Alberdi Collantes \\ Departamento de Geografía de la Universidad del País Vasco
}

\begin{abstract}
RESUMEN
El desarrollo de las identificaciones y

lábeles de calidad de los productos agrarios se convierte en una opción de progreso para aquellas explotaciones agrarias que, no pudiendo incrementar ostensiblemente su capacidad productiva, apuestan por producir un género con unas

características especiales y de calidad,

dirigido a satisfacer la demanda de

pequeños nichos de mercado y obtener así unos rendimientos económicos que les

permitan continuar con las funciones

agrarias. El País Vasco, un medio de montaña con una agricultura insuficiente pero con un mercado urbano importante,

va desarrollando progresivamente

Development of identifications and quality marks of farm products is beginning in an option of progress for different farm exploitations whit structural problems to increase the productions ability. These exploitations development the quality of their productions and get more economic rent ability in the market. Basque Country is a mountain and poor agricultural region but, at the same moment, has a very important urban market in the near proximity and demand quality and original products. Farmers, using quality identifications, are reformer their exploitations and productions to the possibilities that the new market is doing.
\end{abstract} identificaciones que garantizan la calidad

y localidad del producto, generando un mercado específico para los productos de la Comunidad. Como resultado, un grupo cada vez mayor de agricultores adecuan su explotación y producción a las nuevas posibilidades que las identificaciones les están aportando.

PALABRAS CLAVE: País Vasco, horticultura, label, denominación de origen, calidad.

KEY WORDS:

Basque Country, horticulture, label, original identification, quality 
La evolución de la agricultura, al menos desde mediados del siglo xx, se caracteriza por una progresiva modernización y mecanización que tiene por objeto producir más y a unos precios más competitivos. En este proceso la capacidad productiva se concentra en un reducido número de unidades caracterizadas en muchos casos por una intensa empresarialización y capitalización mientras la gran mayoría o abandona las funciones agrarias o inicia una etapa en la que éstas se van marginalizando progresivamente ${ }^{1}$.

En la medida en que las funciones agrarias tienen cada vez menos peso entre la población rural, se hace necesario diversificar la economía de aquellas personas que habitan en este medio y conseguir de este modo mantener a su población. Las actuaciones diversificadoras iniciadas, fundamentadas en la calidad paisajística de los espacios rurales, presentan una marcada orientación hacia iniciativas relacionadas con el turismo hasta el punto de que en muchas comunidades son estas funciones las únicas impulsadas.

A lo largo de los noventa se han ido desarrollando progresivamente otra serie de actividades agrarias que trataban de identificar la calidad natural y paisajística del medio rural con la excelencia de las producciones agrarias tradicionales de muchos de los espacios rurales. Estas iniciativas abogan por una defensa de la calidad, de la originalidad de unos productos y cultivos tradicionales frente a la cantidad que ofrecen las áreas donde la intensificación es más factible.

Sin olvidar la necesaria modernización y mecanización de las unidades productivas se van incorporando diversas iniciativas de comercialización que persiguen alcanzar determinados nichos de mercado en los que el género fresco, de calidad y con un origen especificado es especialmente valorado y en el que se consigue un valor añadido que permite a muchas explotaciones obtener unos resultados económicos satisfactorios. La comercialización sin apenas intermediarios y la diferenciación del producto mediante logotipos de calidad y de origen son también algunas de las posibilidades que ofrecen muchos de estos espacios?

\section{APUESTA POR LA PROTECCIÓN DE LA CALIDAD AGROALIMENTARIA EUROPA Y ESPAÑA}

Uno de los objetivos iniciales de la política agraria común (PAC) era aumentar la productividad agrícola de la Comunidad Europea para conseguir un nivel ele-

\footnotetext{
1 Este trabajo ha sido realizado en el marco de un proyecto de investigación sobre Los productos agropecuarios de calidad en el Pais Vasco, financiado por la Universidad del País Vasco (código 9/UPV00155.130-14524/2002).

2 Como señalan Hernández et al. (2000) las actuaciones diversificadoras de las actividades económicas en el medio rural presentan una marcada orientación hacia las actividades relacionadas con el turismo. Sin embargo, el potenciamiento de las actividades rurales mediante la mejora de los cultivos tradicionales, la difusión de nuevos aprovechamientos, la introducción de mejoras técnicas o la propia pluriactividad agraria puede contribuir a su mantenimiento. Las iniciativas deben abogar por una defensa de la calidad, de la originalidad de estos productos frente a la cantidad que ofrecen las áreas donde la intensificación es más factible.
} 
vado de autoabastecimiento alimentario. Al alcanzarse este objetivo con creces a finales de los años ochenta, la política agraria común comenzó a interesarse en mayor medida por otros como el fomento de los productos de calidad o la integración de la protección del medio ambiente en la agricultura.

La normativa de protección más antigua se ceñia exclusivamente a los productos vitícolas y se remonta a 1970, año en que se constituye una organización común de mercado (OCM) para el sector regulada mediante el Reglamento 816/70. Atendiendo a la descripción que realiza Galdos (2003) será en 1992 cuando la Unión Europea da un paso adelante en la promoción y protección del resto de los productos agroalimentarios al crear los sistemas conocidos como Denominación de Origen Protegida (DOP), Indicación Geográfica Protegida (IGP) y Especialidad Tradicional Garantizada (ETG) ${ }^{3}$. Con la puesta en marcha de estos sistemas se busca fomentar la diversificación de la producción agrícola y facilitar al consumidor información clara y concisa sobre las características y origen de los productos agricolas y alimenticios. Por denominación de origen se entiende el nombre de una región o de un lugar determinado que se emplea para designar un producto agrícola o alimenticio originario de dicha región o lugar y cuya calidad o características se deben al medio geográfico en el que se realiza la producción, la transformación y la elaboración. Por indicación geográfica se entiende el nombre de una región o lugar determinado con el que se designa un producto agricola o alimenticio originario de dicha región o lugar que posea una cualidad determinado o reputación atribuida a dicho origen geográfico en el que se habrá realizado al menos la producción, transformación o elaboración. En ambos casos, DOP e IGP, las definiciones se centran en el vínculo con el territorio, siendo en el primer caso completo (todo el proceso desde la producción hasta la elaboración está ligado al territorio) y en el segundo caso parcial.

España, siguiendo con la trayectoria europea, cuenta con una larga tradición en la promoción de la calidad de los productos agroalimentarios. Según Galdos (2003), la primera protección legal, exclusiva para los vinos, se remonta al año 1932 año en que se aprueba el Estatuto del Vino, que constituye el primer intento de regulación completa del sector.

En 1985 se amplia el campo de las figuras de protección de la calidad con la publicación del Real Decreto 1573/1985 por el que se regulan las Denominaciones de Origen Genéricas y Específicas de productos agroalimentarios no vínicos. Para esta fecha se había producido un acontecimiento fundamental en España como fue la aprobación de la Constitución española de 1978 y se estaba en vísperas de otro no menos importante como era el ingreso en las Comunidades Europeas que se materializa al año siguiente. La Constitución de 1978 configura un

${ }^{3}$ La filosofía de la DOP es elaborar un producto único, no reproducible en otro territorio y susceptible de variar en el tiempo en función de las condiciones climáticas y de la pericia de cada productor. LA IGP se basa por su parte en la reputación del producto, en su historia, ligada a la de una localidad o región, que le proporciona las caracteristicas o cualidades particulares; se apoya en un documento normativo que asegura el mantenimiento de la calidad del producto y no impone una única zona de elaboración del producto (Bérard et al., 2001). 
nuevo modelo de organización territorial, el Estado autonómico, que dio lugar a un sistema de distribución de competencias entre las Comunidades Autónomas y la Administración estatal. Al corresponder a las Comunidades Autónomas las competencias en agricultura ello supuso un profundo cambio en los esquemas de dependencia administrativa de las Denominaciones de Origen. Por otro lado, la integración de España en la actual Unión Europea supone la aceptación de todo el acervo comunitario y el reconocimiento de la supremacía de sus normas sobre el ordenamiento jurídico interno.

La política de promoción de la calidad de los productos agroalimentarios desarrollada a través de la normativa legal descrita ha sido especialmente eficaz en España a la vista de la evolución experimentada por las distintas denominaciones de calidad. Así, por ejemplo, en 1986 las zonas vitivinícolas con Denominación de Origen eran 26 , lo que suponía el $32,2 \%$ del total de la superficie del viñedo dedicado a la transformación; en la actualidad existen 60 zonas amparadas por Denominación de Origen. En la campaña 2000/2001, la superficie inscrita bajo Denominación fue de 641.000 hectáreas (el $56,6 \%$ del total de la superficie dedicada al viñedo para transformación).

Respecto a los restantes productos agroalimentarios la evolución ha sido la siguiente: en 1987 sólo 12 estaban registrados como Denominaciones de Origen y/o Denominaciones Específicas; en 1992, justo antes de la entrada en vigor del Reglamento comunitario, los productos registrados eran ya 26; en el año 2002 se había alcanzado la cifra de 102, diferenciados entre Denominaciones de Origen Protegidas (57 productos), Indicaciones Geográficas Protegidas, (42 productos) y Especialidad Tradicional Garantizada (3 productos), los cuales cubrían una amplia gama de productos de origen vegetal, cárnicos y lácteos.

Además de estas denominaciones reconocidas oficialmente y por tanto incluidas en el registro de la Unión Europea, existen otras muchas, de mayor o menor prestigio, avaladas por las Comunidades Autónomas. A lo largo de los años ochenta y noventa todas las Comunidades Autónomas, haciendo uso de sus competencias, han ido desarrollando políticas de fomento y promoción de la calidad de los productos agroalimentarios con grandes semejanzas entre sí tanto en los mecanismos implantados como en los objetivos.

\section{LA POLITIICA DE CALIDAD EN EL PAISS VASCO}

El Estatuto de Autonomía aprobado en 1981 otorga a las administraciones vascas (Gobierno Vasco y Diputaciones Forales) competencias en agricultura y desarrollo rural, proporcionándoles de este modo la capacidad de iniciativa en la elaboración de una política de calidad agroalimentaria. A partir de entonces un objetivo de las administraciones vascas será el obtener la máxima categoría de calidad para otros productos específicos vascos que gozaban de gran tradición como son el queso y el vino obtenido de las áreas vitícolas vascas de clima atlán- 
tico-húmedo y que se conoce con el nombre de txakoli. Los primeros resultados se consiguen en 1987 año en que se aprueba el reglamento de la Denominación de Origen del queso "Idiazabal", un queso elaborado con leche pura de oveja de las razas latxa y carranzana. En 1990 se reconoce la Denominación de Origen Txakoli de Getaria que abarca una pequeña zona vitícola de la costa de Guipúzcoa; en 1994 llega el reconocimiento de la Denominación de Origen Txakoli de Vizcaya y en el año 2002 de la Denominación de Origen Txakoli de Alava ${ }^{4}$.

Además de los productos amparados por denominaciones de origen, en el País Vasco existen otros a los que se ha concedido un signo de calidad creado por la administración vasca inspirado en el modelo francés (el Label rouge creado en 1960): es el Label vasco de calidad alimentaria el cual se ha convertido en un pilar fundamental de la política de fomento y protección de la calidad de los productos alimentarios del País Vasco. Los motivos alegados (decreto 203/89) para su creación son dos: por un lado, se quiere proporcionar a los consumidores un medio que les permita distinguir los productos cuya calidad supera la media general dentro del conjunto de la oferta alimentaria; y por otro, que los agricultores y elaboradores de productos agroalimentarios de alta calidad puedan disponer de elementos distintivos que les permitan destacarse del anonimato.

El decreto contempla asimismo la creación del Consejo Regulador del Label Vasco de Calidad Alimentaria, organismo integrado en el Departamento de Agricultura del Gobierno Vasco al que se le encomiendan las siguientes atribuciones: analizar las peticiones de integración de los productos en el Label; elaborar los reglamentos específicos de los diversos productos integrados y controlar la calidad de los productos amparados tanto en la fase de producción como en la de elaboración y comercialización. Hasta el año 1998 en que estuvo en vigor esta normativa se habían creado 10 Lábeles de calidad y una marca de garantía de calidad. En 1998 el Gobierno Vasco (decreto 297/1998) procede a derogar las disposiciones normativas ligadas a la creación del Label Vasco de Calidad Alimentaria y adopta un nuevo marco jurídico de gestión para el Label acorde con la legislación europea con lo que se crea la Fundación Kalitatea, un organismo de carácter privado en cuyo consejo directivo participan representantes de la administración vasca y de los productores y que será al que competa la certificación y control de los Labeles vascos de calidad alimentaria, de los reconocidos con anterioridad y de los nuevos que puedan crearse en el futuro. En la actualidad son 11 los productos amparados con el distintivo de Kalitatea de los que 5 son de origen vegetal (Patata de Alava; Pimiento de Gernika; Tomate de calidad del País Vasco; Guindillas de Ibarra y Alubias del País Vasco ) y 6 son de origen animal (Cordero Lechal del País; Poilo de

4 Los recursos agrarios y alimentarios locales y tradicionales, según recoge Berard (1996), llamados productos de la tierra, encarnan en la actualidad las esperanzas de un número cada vez mayor de agentes. Con todo, no se debe de olvidar lo que tienen en común: el hecho de inscribirse en una cultura reciente que se ha promulgado en una reglamentación a nivel europeo. Esta hace referencia a la tradición, el carácter local, el origen y el prestigio y se apoya implicitamente en nociones como la notoriedad, la autenticidad, la tipicidad y las costumbres. 
Caserío; Leche Pasterizada del País Vasco de Alta Calidad; Carne de Vacuno; Miel; Bonito del Norte Fresco ).

La apuesta por un modelo productivo que potencia más la calidad que la denominación geográfica, siguiendo el ejemplo francés, se justifica por la presencia de un tejido agrícola insuficiente pero también por la posibilidad que aporta el mercado desarrollado en la proximidad. Con ello se pretende buscar una salida a un género que se puede acoger a una denominación o identificación de origen protegida pero también a aquellas producciones que, no siendo exclusivas del País, obtienen unos índices de calidad elevados, propios de explotaciones de pequeñas dimensiones.

\section{CULTIVOS TRADICIONALES Y NUEVOS PRODUCTOS COMO OBJETO DEL LABEL DE CALIDAD}

En octubre de 1993 se presenta en Gernika la marca Euskal Baserri, un distintivo que trata de garantizar el origen y calidad de las hortalizas del País Vasco, pensado para que los consumidores identifiquen fácilmente los géneros ofertados por los horticultores del País Vasco. No se trata ni de una denominación de origen ni de un distintivo de calidad sino de una marca identificativa que tiene como objeto diferenciar el producto horticola y frutícola realizado en la Comunidad Autónoma.

El reglamento de uso de la marca Euskal Baserri prevé la utilización de la misma por todos aquellos productores hortofrúticolas que lleven a cabo su labor dentro de la Comunidad Autónoma Vasca. Asimisrno, establece mecanismos de defensa, vigilancia, fomento y control de la calidad del género, que son encomendados a la fundación Kalitatea y al propio Gobierno Vasco.

En realidad, adoptar el nombre Euskal Baserri no es otra cosa que proporcionar un mejor vestido al producto del País ya normalizado ${ }^{5}$. Sólo pueden comercializarse por el mismo hortalizas elaboradas en el País Vasco, sin importar si son vendidas de forma directa o a través de intermediarios. La posesión de la marca se visualiza en una pegatina o similar que porta cada producto unitario o envase, según los casos. El reglamento define las pautas que cada agricultor ha de seguir en cuanto a técnicas de elaboración, almacenamiento, clasificación, envasado e identificación. Euskal Baserri es ante todo un servicio de información al consumidor acerca de la naturaleza y calidad del género ofertado en el mercado.

Sin embargo, el carácter genérico de la distinción Euskal Baserri se muestra insuficiente para distinguir y clasificar determinados productos hortícolas claramen-

5 Como señala el Viceconsejero de Agricultura del Gobierno Vasco en la presentación de la marca Euskal Baserri, ante la avalancha actual de frutas y verduras procedentes del exterior y no siempre de tipología y sabor del producto del País, se hace imprescindible identificar el producto propio, oriundo de la zona. Conscientes de este dato, se pone en marcha el distintivo Euskal Baserri, dentro de lo que definen como política de apoyo y defensa del sector agroalimentario (HAGINA, 1994, p. 24). 
te diferenciados por los consumidores y sobre los que existe una tradición culinaria y de consumo extendida y popular. En estos casos, con el objeto de desarrollar aún más este tipo de producciones, aprovechando la demanda que tienen en el mercado, se opta por desarrollar lábeles de calidad, con su propio sistema de control, diferenciación y garantía de calidad. Son cuatro los productos hortícolas regulados por un Label de calidad: la Guindilla de Ibarra, el Tomate, el Pimiento de Gernika o la Alubia del País Vasco. La personalidad y notoriedad de las campañas de promoción desarrolladas en torno a estos productos, junto al esfuerzo productivo y comercial de los agricultores, contribuye a que se consoliden y se conviertan en una de las producciones principales y características de la horticultura vasca.

El Tomate de calidad del País Vasco obtiene tal calificación en el año 1997 aportado por la fundación kalitatea. Respecto a los caracteres que ha de tener para recibir tal calificación, el fruto ha de ser de forma surcada y entreverada, de color entre verde y rojo. Se distribuye con calibres homogéneos y deberá tener un tamaño siempre superior a $65 \mathrm{~mm}$. Tanto cada tomate como las cajas en las que se comercializan deberán ir acompañadas de su correspondiente etiqueta identificativa. Las variedades aceptadas son Jack, Ramón, Pío, Cabrales y Robin, si bien éstas pueden ir variándose anualmente, en función de las decisiones que la fundación y el comité profesional creado para el control y vigilancia del producto vayan tomando.

El Pimiento de Gernika con Eusko Label consigue la denominación en el año 1993. Respecto a los caracteres que ha de tener para recibir tal calificación el fruto ha de ser de color entre verde medio y oscuro y con un pedúnculo entero, largo y fino. Se distribuye con calibres homogéneos y deberá tener un tamaño entre 6 y $9 \mathrm{~cm}$. Para facilitar al máximo su conservación, protección e identificación se comercializa en envases cerrados de dos docenas, identificados con una etiqueta numerada de control. Las variedades utilizadas son el resultado de un proceso de selección realizado en los caseríos de la zona, aceptándose únicamente dos para el conjunto de productores.

El modo de producción empleado en la obtención de Pimiento y Tomate con Label apenas tiene que ver con el utilizado tradicionalmente. De una producción al aire libre y centrada en los meses de verano se ha pasado a cosechar la mayoría de los meses del año utilizando para ello sistemas productivos que controlan todas las necesidades de la hortaliza.

La Guindilla de Ibarra con Eusko Label obtiene tal calificación en el año 1997. Es un ecotipo especial de la Comunidad Autónoma, con una recolección muy selectiva puesto que solamente consiguen ser producto Label aquéllas de carne tierna y piel fina, de entre $5-12 \mathrm{~cm}$. de longitud y con un pedúnculo estrecho y alargado. Ha de ser un género exento de picor y de color verde amarillento. Por otro lado, sólo se presenta al consumidor en envases de 250 y $425 \mathrm{ml}$, en vinagre de vino poco ácido y con poca sal. El cultivo se alarga desde abril a octubre, con un periodo de producción muy concentrado entre junio y agosto. Se cultiva en matas de mediana de altura, de alrededor de $40 \mathrm{~cm}$., y la recolección y selección del fru- 
to es enteramente manual. A diferencia de los ejemplos anteriores, la producción se realiza al aire libre y sobre sustrato natural siendo muy reducido el empleo de abonos químicos y de tratamiento fitosanitarios. La dependencia de las condiciones climáticas anuales en la producción final es muy elevada en este cultivo

La Alubia con Eusko Label obtiene la calificación en el año 1999. Las variedades autorizadas con el distintivo de calidad Eusko Label se agrupan en tipos diferenciados. La Alubia de Tolosa es de color morado oscuro, de forma ovalada, de piel fina y de excelente mantecosidad. Es un tipo de mata alta, habitualmente asociada al cultivo del maíz. En función de su tamaño y el peso de los granos esta Alubia se denomina txiki si mide entre 0,5 y $1 \mathrm{~cm}$ y cuyo peso de 100 semillas se encuentra entre 35 y $45 \mathrm{~g}$. Haundi se denomina si tiene entre 1 y $1,5 \mathrm{~cm}$ de longitud y un peso comprendido entre 50 y $65 \mathrm{~g}$. Este ecotipo se produce en el área atlántica de la Comunidad Autónoma.

La Alubia de Gernika es de color entre rojo y granate con pintas rosáceas, de forma ovalada o redonda, de piel fina. Es un tipo de mata alta, habitualmente asociada al cultivo del maíz. En función el peso de 100 semillas se encuentra entre 40 y $60 \mathrm{~g}$, en un cultivo que se extiende desde mediados de Mayo hasta finales de septiembre, con una media de 120 a 140 dias. Este ecotipo se produce en el área atlántica de la Comunidad Autónoma.

La Alubia Pinta Alavesa es de color granate con pintas blancas, de forma ovalada o redonda, de piel fina. Es un tipo de mata baja. En función el peso de 100 semillas se encuentra entre 40 y $60 \mathrm{~g}$, en un cultivo que se extiende desde mediados de Mayo hasta finales de septiembre, con una media de 100 a 110 días. Este ecotipo se produce en el área mediterránea de la Comunidad Autónoma.

La localización geográfica de las explotaciones productoras de Alubia con Label está muy extendida, especialmente en la zona atlántica, siendo un cultivo muy habitual en un caserío ganadero, aunque generalmente muy marginal y dedicado al autoconsumo de la familia.

La descripción realizada marca una diferenciación explicita entre el Tomate y el Pimiento, producciones más normalizadas, profesionalizadas e inmersas en una dinámica dirigida a controlar e incrementar la producción y la Guindilla y la Alubia de la zona atlántica, cultivos en los que la renovación técnica ha sido muy escasa, la incidencia climática es importante y en los que el grado de dedicación y profesionalización de sus productores es muy reducida.

\section{FUNCIONAMIENTO SIMILAR PARA UNA DIVERSIDAD DE MARCAS E IDENTIFICACIONES}

El funcionamiento de las identificaciones y lábeles de los cultivos horticolas del País Vasco responde a unos parámetros similares en todos los casos. Por lo general, el Label se aplica a aquellos cultivos de mayor representación y tradición en- 
tre los consumidores, impulsando la consecución de un género normalizado e identificado y creando nuevos canales de comercialización y despacho del producto diferenciado.

La calificación generalmente la realiza el horticultor, que es el que define cuáles son los productos que cumplen las condiciones de calibre, textura y olor que recoge el reglamento. El mismo horticultor etiqueta su género con el identificativo del Label, que combina un diseño gráfico común y un apartado de identificación individual de la explotación. Asimismo, lleva el control de los productos que despacha con la marca. En el caso de aquellos que han de ser manipulados, para los que se necesitan unas condiciones mecánicas y/o sanitarias determinadas, la calificación, preparado y etiquetado se realiza en centros de envasado específicos.

Otro aspecto que presenta unos caracteres comunes estriba en el control que ejerce la Fundación, que se concreta en la realización de visitas en la misma explotación, en los centros de envasado y en los puntos de venta, generalmente en las grandes superficies. Se toman distintas muestras del producto y se analizan generalmente sus caracteres físico-químicos.

El sistema de relaciones que se establece con lo productores y usuarios de la marca también es similar. En la horticultura vasca los agricultores se agrupan en asociaciones profesionales provinciales. A través de ellas se aporta asesoramiento técnico, se gestionan compras de insumos e incluso se ofertan puntos de venta. Estas asociaciones tienen su propio reglamento de funcionamiento, órganos de gestión y de representación e incluso sus propios presupuestos, colaborando de manera activa con la administración.

Las asociaciones de horticultores, sin embargo, no toman parte en la constitución de la fundación Kalitatea y su relación con los lábeles de calidad del área vegetal es muy reducida. Por lo general se concreta en la puesta en marcha de algún proyecto de investigación, en la compra de insumos o fitosanitarios específicos o en la colaboración en el asesoramiento técnico al productor que despacha utilizando las identificaciones aunque siempre de un modo puntual y sin intervenir ni en la gestión, diseño y comercialización del producto Label.

La participación de los agricultores en el diseño del género que van a clasificar se concreta en el Comité Profesional de cada uno de los lábeles, que cuenta con una representación de agricultores, en el que se discuten aspectos relativos a la producción (fitosanitarios), variedades e incluso al marketing de la marca. Sin embargo, éste es un órgano consultivo, que por lo general se reúne una vez al año y es la propia fundación la que toma las decisiones principales respecto al futuro de la marca.

La fundación Kalitatea, además de ser una entidad certificadora de la calidad del producto, desarrolla unas marcas de su propiedad, con unas denominaciones específicas y un género que se controla desde su cosecha hasta su venta. A diferencia de otras denominaciones y lábeles de calidad, en los que los productores y 
envasadores dirigen la evolución y exigencias de las marcas, en el ejemplo estudiado su participación es muy reducida y es la fundación la que decide el funcionamiento de la marca ${ }^{6}$. Si la fundación nace con el objetivo de certificar y controlar la calidad de los productos con Label, como hemos comentado, actualmente no limita su función a las labores de acreditación sino que asume la responsabilidad de planificar y gestionar cada uno de los identificadores y con ello, parte de las posibilidades de desarrollo del sector.

\section{HACIA LA CONSECUCIÓN DE UN HORTICULTOR QUE SÓLO PRODUCE CALIDAD}

Todos los cultivos hortícolas identificados son productos tradicionales de la huerta vasca, algunos de ellos son de tradición centenaria, como es el caso de la Alubia de Tolosa o el Pimiento de Gernika. Otros tienen una implantación más reciente, como la Guindilla, cuya presencia comienza a constatarse a principios del pasado siglo y en alguno más, como ocurre con el tomate, han sido sustituidas las variedades tradicionales por otras más productivas y adecuadas a la demanda del consumidor.

La tipología de explotación hortícola que encontramos en el País Vasco-Atlántico ha tendido generalmente a producir una gama amplia de cultivos. Esta tendencia tiende a variar en la última década en la que cada vez más es mayor el número de horticultures que se especializan en un único cultivo. Por lo general el mayor grado de dedicación, profesionalización, formación e incluso continuidad en las funciones agrarias lo localizamos entre aquellas explotaciones que producen Tomate y Pimiento de Gernika, cultivos que muestran los mayores índices de tecnificación del sector y los mejores resultados económicos de todos los productos de la huerta vasca. Al mismo tiempo, son los productos que comercializan mayor número de unidades utilizando el Label vasco de calidad.

El Pimiento de Gernika despachado con el anagrama de Eusko Label presenta un crecimiento continuado en docenas comercializadas, multiplicando por diez el número de unidades producidas desde 1993 al 2001 y ello teniendo en cuenta que en el mismo periodo el número de productores registrados ha pasado de 16 a 34 . Este incremento por unidad productiva está relacionado con la progresiva incor-

${ }^{6}$ Es bajo estas condiciones estructurales y productivas por lo que se decide apostar por una política de calidad al entender que es una de las pocas herramientas que disponen zonas como el País Vasco para lograr que sus principales producciones agroalimentarias sean competitivas. Los mayores esfuerzos se han centrado en la creación del Label de calidad alimentaria (la agricultura ecológica en el País Vasco está poco desarrollada y la Producción Integrada carece de reglamentación) para lo cual se ha seguido el modelo francés pero con una diferencia: mientras que en Francia la iniciativa de crear el Label fue impulsada por el propio sector agrario, en el País Vasco es la propia administración regional la que lidera el proceso, teniendo para ello que acometer la doble tarea de implicar a un sector productivo poco organizado y poco acostumbrado a someterse a controles de calidad y seguridad alimentaria, y de informar a los potenciales consumidores (Arruti, 1999). 
poración de nuevos invernaderos con la tecnología más avanzada, producción sin suelo e instalaciones para la conservación y manipulado del Pimiento, aumentado con todo ello la rentabilidad de este cultivo.

Tabla 1. Evolución de la producción y del número de productores de Guindilla con Label

\begin{tabular}{lccccccccc}
\hline & 1993 & 1994 & 1995 & 1996 & 1997 & 1998 & 1999 & 2000 & 2001 \\
\hline Productores & 16 & 30 & 30 & 30 & 58 & 59 & 56 & 44 & 34 \\
\hline Envasadores & 3 & 3 & 5 & 8 & 8 & 9 & 9 & 9 & 9 \\
\hline Docenas Label (miles) & 78 & 225 & 236 & 250 & 366 & 598 & 661 & 696 & 738 \\
\hline
\end{tabular}

Fuente: Fundación Kalitatea. Año 2000.

Tabla 2. Evolución de la producción y del número de productores de Tomate con Label

\begin{tabular}{lccccc}
\hline & 1997 & 1998 & 1999 & 2000 & 2001 \\
\hline Productores & 4 & 54 & 41 & 40 & 53 \\
\hline Envasadores & 4 & 13 & 17 & 20 & 29 \\
\hline Kilos Label (miles) & 18 & 225 & 451 & 593 & 1.120 \\
\hline
\end{tabular}

Fuente: Fundación Kalitatea. Año 2000.

Similar evolución ha presentado el cultivo del Tomate con Label aunque en este caso se ha de destacar la intensidad que el crecimiento de agricultores y de kilos registrados está teniendo, animados por una buena respuesta del consumidor y por la consecución de un mayor valor añadido. Así, entre los años 2000 y 2001 se ha duplicado la producción, mientras el número de productores pasaba de 40 a 53. En la consecución de estos resultados hemos de situar la tendencia de muchas explotaciones y, especialmente de aquéllas de nueva incorporación, de dirigir su equipamiento y tecnología a producir exclusivamente Tomate.

La explotación especializada en estos cultivos, según un estudio de la consultoría Gatik (2002) relativo a los horticultores vizcaínos, marca algunas diferencias importantes respecto a la unidad hortícola tradicional. Ésta última se caracteriza por el predominio de la persona física como forma exclusiva de explotación, de un titular que en el $85 \%$ de los casos supera los 55 años, con una continuidad en la explotación familiar garantizada en el $10 \%$ de los ejemplos y que dedica a cultivos hortícolas una media de $4.511 \mathrm{~m}^{2}$, de los que el $75 \%$ se destinan a cultivos al aire libre que combina con la producción en túneles de plástico y sobre suelo natural preferentemente de lechuga pero también de Tomate y Pimiento de Gernika. Entre las tipologías de explotación entre las que la horticultura adquiere cierta relevancia 
económica y de dedicación es ésta la más numerosa, con un total estimado para el conjunto provincial de 654 explotaciones. Su utilización de la marca, sin embargo, es muy reducida y tan sólo el $7 \%$ de ellas, asociadas a la cooperativa de comercialización Garaia, despachan con el distintivo.

La explotación hortícola que utiliza los distintivos de calidad, al igual que la tradicional, coincide básicamente con la unidad agraria vasco-atlántica, el caserío, aunque en este grupo predominan titulares de edad más joven, alrededor de la mitad inferior a 40 años, que dirigen la explotación como personas físicas pero también desarrollando otro tipo de personalidades jurídicas asociativas. Sin embargo, en número de explotaciones son menos numerosas que las anteriores (146 en el territorio de Vizcaya) aunque el nivel de formación, la superficie dedicada a cultivos hortícolas por explotación $\left(5.700 \mathrm{~m}^{2}\right)$ y sobre todo la superficie cubierta por invernaderos ( $86 \%$ del total del cultivo hortícola) es sensiblemente superior. Se caracterizan por la alta especialización en tres cultivos: lechuga, Pimiento verde y Tomate, siendo habitual que tan sólo desarrollen una producción o que la combinen con una segunda (Tomate-Pimiento; Tomate-Lechuga; Pimiento-Lechuga). Por lo general, la lechuga se produce sobre suelo cubierto por invernadero tipo túnel o bitúnel, mientras en Pimiento y sobre todo Tomate comienza a dominar el cultivo hidropónico sobre multitúnel de paredes verticales ${ }^{7}$. Fertirrigación automática, sistemas de calefacción, ventilación automática, pantalla térmica..., son algunos de los equipamientos presentes en un buen número de estas explotaciones. A pesar de que el número de productores y la superficie dedicada sea sensiblemente menor que la del grupo tradicional, en producciones como el Pimiento, Tomate o lechuga llegan a concentrar entre el 55 y el $75 \%$ del valor económico total de estos cultivos en la provincia ${ }^{8}$. Del total de la mano de obra utilizada, el $59 \%$ es familiar, siendo los asalariados fijos el $9 \%$ y los eventuales el $32 \%$.

La tipología de explotación presentada es la que recurre de manera masiva a la utilización de la identificación Euskal Baserrio al empleo de los lábeles de calidad y lo hace en la mayoria de los casos agrupándose en una cooperativa, Garaia, responsable de la comercialización del total de la producción de los horticultores socios. Desarrollan una unidad horticola cuyo objetivo prioritario es incrementar la producción por unidad de superficie y dejan en manos de terceros el envasado, etiquetado y despacho de la producción. En la medida en que el volumen a comercializar es considerable, Garaia apuesta por despacharlo en grandes centros de

7 Son los invernaderos multicapilla, de placa, los que mayor desarrollo están presentando en Guipúzcoa en los últimos años (HAGINA, n. ${ }^{\circ} 13,1997$, p. 23). Sobre este tipo de invernaderos NEBOT, gerente de Invernaderos e Ingeniería S. A. (ININSA), señala que «Esta evolución de estructuras y cubiertas que hacen a los invernaderos: multitúnel tipo industrial, equiparables a los de vidrio en cuanto a la posibilidad de control de clima interior, ha hecho posible, además, dotarles de instalaciones interiores similares o iguales a las utilizadas en los invernaderos de vidrio y conseguir, por tanto, unas producciones equiparables" (HORTICULTURA, n. ${ }^{\circ}$ 100, 1995, p. 41).

${ }^{8}$ La producción de Pimiento y Tomate con hidroponia y con invernadero climatizado estaba aportando en el años 2002 un valor económico de $30,05 / \mathrm{m}^{2}$. Las misma producción en un invernadero sin control climático y sobre sustrato natural aportaba un valor de 12,2/ $\mathrm{m}^{2}$. (GATIK, 2002). 
venta como es el mercado al por mayor de la provincia, Mercabilbao, o los distintos hipermercados y cadenas de distribución. La identificación sustituye a la relación personal que desarrollaba el horticultor tradicional utilizando las marcas en la totalidad de la verdura comercializada.

Los horticultores guipuzcoanos, menos numerosos que los vizcaínos en cuanto al número de registrados a la marca (4 en Pimiento y 18 en Tomate), responden a los parámetros productivos y a la evolución que hemos observado en Vizcaya. Generalmente se trata de unidades que coinciden con el caserio tradicional, una explotación que combinaba horticultura y vacuno. Hoy en día sigue manteniendo este carácter familiar si bien se ha abandonado el ganado y es la horticultura en invernadero la principal actividad de estas explotaciones. Las producciones principales se corresponden con las más habituales en la huerta del País Vasco, tomate, vaina y lechuga, junto a la producción a otro nivel de berzas, berenjenas, puerros, etc. Se trata de una explotación media, que posee entre 3 y 5 mil metros en invernadero y otros tantos cultiva al aire libre, superficie suficiente como para emplear a tres personas a jornada completa en esta actividad. Cuenta además con alrededor de $3 \mathrm{Ha}$. de mayor pendiente y adecuadas a usos herbáceos.

Entre las particularidades que presenta cabe señalar el mantenimiento de una producción más variada que la vizcaina, justificada por haber desarrollado un sistema de comercialización individual y dirigido al mercado de la proximidad. Sin embargo, también en este territorio el sistema de comercialización se está concentrando y paralelamente el agricultor tiende a especializarse en unos pocos cultivos, incorporando mejoras técnicas que aseguren e incrementen la cosecha. Una muestra de ello es el desarrollo de polígonos hortícolas, áreas adecuada con dinero público y dirigidas a horticultores de nueva incorporación, destinadas a cultivos en invernadero y cuyas explotaciones están desarrollando en la mayoria de los casos un único producto, el Tomate, identificado con Label y dirigido al mercado al por mayor y a la gran superficie. La diferencia principal se establece en el sistema de comercialización, realizado individualmente por el horticultor aunque se tiende a despachar en unos pocos puntos de referencia ${ }^{9}$.

Si el tipo de explotación que comercializa Tomate, Pimiento verde e incluso Euskal Baserri se caracteriza por su alto grado de profesionalización y dedicación

9 Esta es la peculiaridad del sector hortícola de Guipúzcoa, es decir, contar con un mercado propio en el que el producto que elabora es apreciado. En un ámbito similar, como Cantabria, al no tener un centro amplio de consumo en la proximidad, han de competir en el exterior con otras zonas productoras para lo cual es primordial la existencia de una estructura de comercialización conjunta. Esta situación provoca adecuarse a una dinámica de precios en la que el mercado ha sufrido a lo largo de este tiempo una serie de altibajos a los que no han podido hacer frente todos los agricultores (HAGINA, n. 9, 1996, p. 35). Incluso Vizcaya no tiene tan desarrollado este tipo de venta, según se deduce de lo que RUIz URRESTARAZU y GALDOS señalan: "El mayor volumen de transacción se realiza del horticultor al mayorista. En la capital vizcaina las ventas se cristalizan en Mercabilbao cuyos mayoristas no compran en firme sino que retienen el $10 \%$ de las ganancias de las ventas producidas, yendo el resto al horticultor... Paralelamente subsiste con mucha menor incidencia la venta tradicional en los mercados semanales de ciertos núcleos de población. Apenas existe venta directa a las tiendas» (1987, p. 232). 
y por su tendencia a especializarse amparándose en la demanda que en el mercado están teniendo estos productos, hasta el punto de ser el referente de futuro de la horticultura vasca, las identificaciones de la Guindilla y de la Alubia no están generando una especialización en estos géneros ni un efecto difusor de su actividad materializado en la incorporación de nuevos agricultores. Ambos cultivos se caracterizan por su sistema productivo tradicional, excesivamente dependiente de las inclemencias climáticas y realizados por un horticultor que no vive de las aportaciones de su cosecha.

En el label de la Guindilla, por ejemplo, entre los 65 productores registrados en el año 2001 tan sólo se cultivaron $10 \mathrm{Ha}$., una media de $1.500 \mathrm{~m}^{2}$ por explotación. Es más, salvo en media docena de casos que combinan este cultivo con otras producciones horticolas, el resto apenas mantiene otra función agraria y su fuente de ingresos prioritaria es urbana. Las aportaciones netas de esta actividad por propietario son inferiores en la gran mayoría de las explotaciones a los 3000 euros anuales. La única excepción la protagonizan tres horticultores que combinan la producción de Guindilla con la gestión del centro de envasado pero su importancia no estriba tanto en la producción obtenida sino en la comercialización del género que recala en el centro.

La Alubia responde a unos indicadores similares e incluso más marginales. A pesar de la extensión de este cultivo en la mayoría de los caseríos vasco-atlánticos, en el año 2001 tan sólo un productor envasó con el nombre de Alubia de Gernika, 9 lo hicieron con el de Pinta Alavesa y la Alubia de Tolosa, la de mayor prestigio en el mercado, contó con 42 productores distintos. Si tomamos esta última como referencia observamos que desde que se creo la asociación a la que posteriormente se prima con el Label de calidad no se han producido nuevas incorporaciones ni ha aumentado la superficie dedicada ni se ha incrementado una producción que se mantiene estanca entre 17.000 y $20.000 \mathrm{~kg} / \mathrm{año}$. De hecho, en el

Tabla 3. Evolución del n. ${ }^{\circ}$ de productores de Alubia de Tolosa en función de su producción (Kg.)

\begin{tabular}{cccccc}
\hline & 1997 & 1998 & 1999 & 2001 & 2002 \\
\hline $0(\mathrm{Kg})$ & & & & 4 & 6 \\
\hline$<100$ & 5 & 1 & 2 & 3 & 6 \\
\hline $100-300$ & 29 & 14 & 11 & 12 & 12 \\
\hline $300-500$ & 10 & 10 & 10 & 15 & 14 \\
\hline $500-1000$ & 7 & 2 & 9 & 7 & 8 \\
\hline $1000-3000$ & 4 & 3 & 2 & 4 & 2 \\
\hline TOTAL PROD & 55 & 30 & 34 & 45 & 48 \\
\hline
\end{tabular}

Fuente: Asociación de productores de Alubia de Tolosa. Año 2003- 
año 2002 se obtuvo una media de $410 \mathrm{~kg}$ por productor, de los que aproximadamente la mitad se comercializaron con el identificativo de calidad y el resto se dedicó a autoconsumo. Si tenemos en cuenta que por cada $\mathrm{kg}$ de Alubia de Tolosa el centro viene a abonar alrededor de 4 euros, estas explotaciones están obteniendo un rendimiento bruto de 800 euros anuales, unos resultados indicativos de la marginalidad económica del cultivo. Es más, excepto dos, todos los productores obtuvieron una cosecha inferior a $1000 \mathrm{~kg}$. La tipología de asociado mayoritario la componen generalmente mujeres próximas a la edad de jubilación y que continúan manteniendo una producción que en estos caseríos ha estado generalmente dedicada a autoconsumo. Las expectativas de producción tampoco son halagüeñas, puesto que se observa que los jóvenes no muestran interés en continuar con la labor de sus padres.

Es difícil, por tanto, valorar la incidencia que las identificaciones y los lábeles están teniendo en el desarrollo de la huerta vasca. Los datos presentados varían excesivamente de un cultivo a otro aunque todo evidencia que los cambios en la dieta alimenticia, la demanda de géneros hortícolas y el incremento generalizado de los precios está contribuyendo a la consolidación de un subsector hortícola en el agro vasco. Los identificativos de calidad ayudan a crear nichos de mercado en los que el producto local obtiene aún mejores resultados, permitiendo a la pequeña explotación sobrevivir en un comercio acaparado por la gran empresa productora y distribuidora.

La principal particularidad estriba en el modelo de explotación hortícola que se está desarrollando, una unidad agraria que apuesta por producir más sin aumentar la superficie utilizada, que se especializa en la obtención de uno o dos productos, siguiendo las indicaciones que establece el reglamento del Label de calidad y que distribuye cada vez en un menor número de puntos de venta o que deja la comercialización de su cosecha en manos de asociaciones o particulares.

Por otro lado, se ha de reconocer que las identificaciones no varian la dinámica de un sector envejecido e insuficiente, que sigue una evolución progresiva hacia la marginalidad de las funciones, a pesar de la buena dinámica de precios y de la labor indiscutible de las marcas. Este aspecto se refleja en la evolución de la horticultura tradicional o en la dificultad de crear un subsector a partir de unos cultivos ya marginales en la huerta vasca.

\section{LA COMERCIALIZACIÓN, EL GRAN ÉXITO DE LAS IDENTIFICACIONES DE CALIDAD}

Los sistemas de comercialización que actualmente están desarrollándose se fundamentan en la concentración de la distribución y la venta en unas pocas firmas comerciales. La pequeña explotación tiene importantes dificultades de adecuación a las exigencias de género normalizado, a la política de precios y a las condiciones de servicio que imponen estos centros. 
Por ello, uno de los objetivos principales de la Fundación es el de responder a la necesidad de adecuar el sector a esta nueva realidad comercial, aprovechando el interés cada vez más evidente de los consumidores por los productos diferenciados y de calidad, que se materializa en la capacidad de crear nichos concretos de calidad.

La horticultura en el País Vasco ha sido tradicionalmente un género de segundo orden en un caserío básicamente ganadero, con una cosecha que era despachada en el mercado diario o en pequeñas tiendas de alimentación. A pesar de los cambios evidentes en los sistemas de comercialización actuales todavía pervive este sistema de venta, incluso entre aquellas explotaciones que han optado por desarrollar funciones hortícolas frente a las ganaderas. Por ejemplo, respecto al sistema de comercialización de los horticultores profesionales de Guipúzcoa (GILBE), según un estudio realizado por la propia asociación en el años 2002 , el $76 \%$ del género producido es vendido en los mercados diarios (33\%) o repartido directamente en tiendas y comercios (43\%). Tan sólo un $13 \%$ es entregado en hipermercados y un $11 \%$ entrega la cosecha a los mayoristas. El sistema de venta sin apenas intermediación sigue siendo el prioritario entre esta tipología de agricultores.

La aparición en el mercado de Euskal Baserri comienza a marcar un punto de inflexión respecto a este sistema de comercialización. Las ventajas que supone tener una marca común para la verdura del País se fundamentan en la identificación que el consumidor del Pais Vasco tiene del género local como sinónimo de calidad y en la posibilidad de diferenciar y promocionar una única marca y un distintivo de calidad en el mercado. Gracias a ello, el número de productores acogidos a la marca se ha ido incrementando año a año si bien todo indica que éste tiende a estabilizarse y son ahora las unidades de producto comercializadas ${ }^{10}$ las que experimentan un mayor aumento.

La promoción publicitaria realizada se concreta generalmente en una difusión mediante distintos medios de comunicación y en los propios puntos de venta, con el objeto de hacer llegar al consumidor las características de la marca y darle a conocer sus distintivos. En este sentido, se advierte que ni todos los centros de comercialización son objeto de dichas campañas ni el interés de los comerciantes es siempre el mismo. En términos generales se observa que es la gran superficie la destinataria de las campañas y también de gran parte del género comercializado mediante los distintivos de origen y calidad. La justificación de comercializar mediante la identificación de origen o el Label de calidad se fundamenta en el interés de los responsables de las grandes superficies por incluir en sus ofertas no sólo productos competitivos en precios sino también en calidad y en localidad.

Las explotaciones que optan por comercializar toda su producción mediante Euskal Baserri se corresponden generalmente con unidades productivas especia-

10 Entre 1998 y el 2000 el número de productores que comercializan con Euskal Baserri pasa de 107 a 115, mientras las unidades comercializadas se incrementan en un tercio en el mismo periodo (HAGINA, 2001, n. ${ }^{\circ} 29$, p. 41 ). 
lizadas en uno o dos productos y que despachan su cultivo en grandes superficies de consumo. Es en este tipo de establecimientos donde la diferenciación del género mediante la marca de origen y calidad adquiere, al menos por el momento, su máxima referencia siendo muy reducido el porcentaje distribuido tanto en mercados locales como mediante minoristas ${ }^{11}$. Este hecho se fundamenta en la menor relación que existe en este tipo de comercios entre el agricultor, distribuidor y consumidor, en la que la etiqueta actúa como sustituto de esta interacción.

La comercialización del género con Label introduce ligeras modificaciones en el sistema habitualmente utilizado en Euskal Baserri. Tomando como referencia el cultivo del Tomate, observamos como en primer lugar es calificado teniendo en cuenta calibres, color y textura y utilizando sistemas manuales. Posteriormente cada Tomate es identificado con la pegatina genérica, que a la vez tiene un pequeño recuadro en el que se recogen los datos de la procedencia de ese Tomate. Antes de ser introducido en cajas de fruta y hortaliza cada una de ellas se recubre con un faldón indicativo de la marca. Las cajas pueden ser de distinto tamaño pero generalmente oscilan entre 10 y $17,5 \mathrm{~kg}$. El resto de productos con Label, bien a modo individual o mediante el centro de el centro de envasado, han de cumplir con un proceso similar. El proceso de selección e indicación no es complicado pero requiere un largo periodo de tiempo y un coste adicional que puede incrementar el coste de la producción en un $20 \%$.

Es difícil calcular si los costos añadidos al proceso de producción son posteriormente recuperados pero todo indica que, al menos en el caso del Tomate, no se abona más por el calificado con Label. Lo que resulta evidente es que la identificación de la calidad y origen del género abre vías de comercialización diferentes, especialmente en hipermercados, en los que se comercializa alrededor del $25 \%$ del Tomate con Label pero también en los centros de venta al por mayor de Vizcaya y Guipúzcoa, donde se llega a comercializarse hasta un $70 \%$ del Tomate producido.

Resulta complicado conocer cuáles son los aspectos que el consumidor valora a la hora de elegir un producto que habitualmente dobla en precio a los otros que se exponen en el mismo stand. Se ha de considerar la procedencia como garantía de calidad o tal vez el sabor del producto. Lo que parece evidente es que aspectos como el color o la forma no parecen haber influido decisivamente. Todo indica que la identificación ha aportado al género un reconocimiento de origen, que a su vez estaba relacionado con la calidad. Ello ha coincidido además con un ciclo

11 Atendiendo a las apreciaciones de una veintena de productores que comercializan utilizando estas distinciones, según recoge el equipo redactor de la revista Hagina en 1997, la gran mayoria de ellos la utilizaban para comercializar en uno o dos puntos de venta, generalmente grandes superficies comerciales. Tan sólo cinco productores la usan en establecimientos de pequeñas dimensiones e independientes de las grandes cadenas de distribución. De hecho, algunos de ellos se quejaban de la escasa aceptación que la marca estaba teniendo por parte de este tipo de establecimiento e incluso del consumidor que habitualmente acude a comprar directamente al productor al mercado de abastos local. La justificación de comercializar mediante la denominación de origen o el Label de calidad se fundamenta en el interés de los responsables de las grandes superficies por incluir en sus ofertas no sólo productos competitivos en precios sino también en calidad y en localidad. 
económico favorable y un cambio en la dieta del consumidor, en la que adquieren un lugar preferente las hortalizas.

En el caso del Pimiento de Gernika, además de los aspectos reseñados, la apuesta por un Pimiento de reducido tamaño y envasado en red ha creado un producto enormemente comercial y el diseño elegido ha sido el adecuado para acceder a nuevos sectores de demanda. El hecho de que la docena de Pimiento verde (alrededor de $300 \mathrm{~g}$.) se venda al consumidor a 4 euros se justifica por el nivel de aperitivo que ha conseguido este producto.

La coincidencia de aspectos positivos, reflejada en la demanda y el precio que el género adquiere en el mercado, está provocando una progresiva especialización de los horticultores en estos cultivos hasta el punto de que un buen número de ellos sólo produce Pimiento o Tomate o ambos respectivamente. Especialmente importante es su incidencia en la incorporación de nuevos agricultores, que en todos los casos apuestas por utilizar marcas identificativas y entre los que el cultivo del Pimiento o Tomate son los preferentes o exclusivos.

El Label en la medida en que está aportando opciones comerciales está teniendo un importante efecto difusor, no tanto en la captación más explotaciones, cuyo número es reducido y permanece estancado, sino en la génesis de modelos productivos especializados en producir Label. La principal diferencia que se establece entre el horticultor tradicional estriba no tanto en qué hortalizas produce sino en cómo las produce y sobre todo en cómo las despacha. Así, tomando como referencia al horticultor vizcaíno, observamos que el tradicional continúa despachando el Pimiento o el Tomate en el mercado local, en pequeñas tiendas o en el mercado de abastos, cuando el grupo más especializado despacha en grandes superficies y en cadenas de supermercados ${ }^{12}$, recurriendo a grupos y asociaciones especializados en la comercialización.

En el caso de la Guindilla y la Alubia, el desarrollo de los lábeles de calidad no ha supuesto cambios importantes en la producción y la extensión de estos cultivos. Desde la creación del Label de la Guindilla el número de centros de envasado se mantiene estanco, al igual que el número de productores, si bien la publicidad del Label está consiguiendo que el consumo de este producto obtenga un mayor precio y se extienda al conjunto del territorio de la Comunidad e incluso a puntos de venta de otras zonas de España. La ampliación del mercado, visible en el caso de la Guindilla, es extensible al resto de productos Label que, con la homogenización y promoción del género, amplian su mercado al conjunto del territorio ${ }^{13}$.

12 Atendiendo a los datos aportados por la consultora GATí (2002), el sector hortícola más profesionalizado despacha prácticamente todo su producción a través de 3 canales diferentes: Mercabilbao, Grandes superficies y Cadenas de supermercados. Frente a ellos, el tradicional utiliza también Mercabilbao pero recurre preferentemente a los mercados locales y a la venta directa al consumidor final.

13 A pesar de la ampliación de mercado al conjunto de la Comunidad Autónoma, hoy en día el mercado local y preferentemente el provincial es el principal desarrollado. Los horticultores Vizcainos, según la consultora GATIK (2002), comercializan en la provincia el 75\% de la producción, un $7 \%$ en otras Comunidades y el resto entre Guipúzcoa y Alava. Aun así, como ocurre con el ejemplo del Pimiento, resulta curioso que la calificación Eusko Label al producto haya tenido mayor fuerza en los nuevos mercados que en el tradicional (Fundación Kalitatea, 2002). 
En el ejemplo de la Alubia de Tolosa todo indica que la identificación no ha aportado al producto más reconocimiento que el que ya tenía entre los consumidores. La Alubia de Tolosa se vende por su propio nombre y no tanto por su promoción con el identificativo de calidad. Sin embargo, el Label ha aportado nuevas vías de comercialización, ha extendido el consumo del producto, persigue la incorrecta utilización del nombre de la Alubia de Tolosa y sobre todo ha potenciado la creación del único centro de envasado existente.

Tanto la Alubia como la Guindilla cuentan con importantes posibilidades comerciales por explorar pero se enfrentan a la ausencia de género durante alrededor de la mitad del año. La tipología de agricultores que cultivan estos productos y la apuesta de los profesionales por otras variedades que requieren menos mano de obra y que están obteniendo un alto precio en el mercado dificulta el desarrollo de unos cultivos con un mercado potencial importante.

Las identificaciones de origen y calidad están aportando al género local la posibilidad de promocionarlo, identificarlo y adecuarlo a un mercado selectivo, hasta el punto de convertirlo en un aperitivo e incluso en el plato preferente de un día festivo, con un consumidor dispuesto a pagar más por un género que ofrece calidad y localidad.

Asimismo, está creando cauces de desarrollo para el subsector hortícola vas$\mathrm{co}$, tanto comerciales como productivos, con unas perspectivas de crecimiento importantes, cuyo freno principal estriba en la dificultades que el reducido número de horticultores tiene para hacer frente al reto comercial que el Label le plantea.

\section{LA IDENTIFICACIÓN COMO HERRAMIENTA BÁSICA EN LA CONSECUCIÓN DE UN SECTOR PROFESIONAL.}

Las explotaciones hortícolas del Pais Vasco responden a una tipología similar, relacionada con el caserío. Son unidades agrarias fundamentadas en las posibilidades que aporta la mano de obra familiar y con una heredad que presenta un extensión y unas posibilidades productivas parecidas en la mayoría de los casos. Así, se observa como se ha evolucionado de un caserio de base ganadera a una especialización hortana, a la que se le destinan aquellos suelos de la heredad que presentan condiciones adecuadas para estos usos, que no son muy extensos en el caserío, dedicando a usos horticolas alrededor de 1,5 $\mathrm{Ha}$. por explotación, de la que entre 3000 y $5000 \mathrm{~m}^{2}$ estarían cubiertos por invernaderos. A pesar de la reducida superficie dedicada el número de personas y el grado de dedición a estas funciones es reseñable, en la mayoría de los casos entre dos o tres personas a tiempo completo.

El modelo de horticultura desarrollado se fundamenta en la proximidad de la ciudad y las explotaciones se sitúan a apenas varios kilómetros de los centros de venta. Es por tanto una agricultura propia de espacios periurbanos, aspecto que 
queda reflejado en el sistema de distribución directa y en las propias características productivas de las explotaciones, normalmente de pequeña dimensión, con una importante presencia de mano de obra y con unos cultivos destinados a satisfacer diariamente el mercado urbano.

La justificación de la presencia de una tipología de explotación reducida, incapaz de competir en producción y costos con las grandes zonas productoras del sur de España, se fundamenta en el desarrollo de unas variedades y un sistema de comercialización sin apenas intermediarios y en el que el horticultor expone un género fresco, que es identificado por parte del consumidor como de gran calidad y por el que está dispuesto a abonar un mayor precio. La importancia de la venta directa ha sido fundamental en el desarrollo de una horticultura competitiva. La posibilidad de diversificar canales de comercialización, desde la venta directa en el mercado a su salida a partir del comercio minorista, ha abierto un abanico de opciones que ha permitido ampliar la cuota de negocio de muchos de ellos.

El desarrollo de las denominaciones de origen y los lábeles de calidad en las producciones hortícolas es un nuevo paso en el progreso de los medios de distribución y venta del producto local. El hecho de amparar la verdura local bajo una identificación común que regule los caracteres físicos y químicos del género según las exigencias del mercado, que gestione logotipos e identificaciones comunes y que realice campañas de promoción de la marca aporta al horticultor la posibilidad de acceder a los nuevos sistemas de comercialización que se están imponiendo estos últimos años. La marca, asimismo, apoyada en la promoción realizada, extiende el ámbito territorial en el que el producto era tradicionalmente ofertado aumentando las posibilidades de venta.

Por otro lado, la delimitación de unos parámetros de calidad y la obtención de un producto altamente valorado permite acceder a nichos de mercado en los que se obtienen unos resultados que multiplican el precio de venta del producto medio. La hortaliza con Label adquiere el carácter de aperitivo o entrante y la legumbre el de plato principal de un día festivo.

La obtención de unos rendimientos más elevados y la extensión del mercado de los productos con denominación, apoyados por el incremento general que está teniendo el consumo y los precios de las hortalizas, está impulsando la consecución de un subsector hortícola profesional en el País Vasco. Aunque la gran mayoría de los horticultores tradicionales carecen de continuidad al frente de la explotación, otro pequeño grupo ha apostado por la tecnificación progresiva de la unidad agraria y por la especialización en la producción de aquellas hortalizas que mayores resultados económicos están aportando en el mercado, especialmente Tomate y Pimiento. Los resultados que están obteniendo no pasan desapercibidos y nuevos agricultores comienzan a instalarse en la horticultura vasca. El identificativo de calidad es fundamental para que el nuevo agricultor o aquél que incrementa su producción pueda obtener la cuota de mercado necesaria para despachar su cosecha y lo pueda hacer a unos precios adecuados. 
El Label, por tanto, está dando nuevas opciones de comercialización al género y al productor, oportunidades que no suponen la erradicación de las que ya tenía en el comercio local y minorista y que incrementan las posibilidades de consecución de un subsector hortícola competitivo. La principal debilidad estriba en las expectativas generadas entre el consumidor, especialmente por determinados productos que rápidamente desaparecen de los lugares de venta por falta de unidades. En algunos casos es el propio horticultor el que no acompaña a los resultados que la marca puede aportar. De hecho, el sector profesional que se está desarrollando es inferior a las 150 unidades en el conjunto del territorio y la superficie cubierta no supera las $50 \mathrm{Ha}$.

El futuro de las identificaciones depende de las posibilidades del sector. El interés de comerciantes y consumidores es manifiesto, los precios obtenidos superan con creces a los del mismo género sin identificación, el mercado contínúa extendiéndose pero el número de horticultores parece haberse estancado, la incorporación de nuevos agricultores es reducida y muy selectiva y a pesar de que el número de unidades comercializadas continúa incrementándose también tiende a estabilizarse. El futuro de los lábeles de calidad dependerá de la intensidad de la renovación del sector hortícola y de la incorporación de nuevas explotaciones.

\section{BIBLIOGRAFIA}

AlBeRDi, J. C. (2001): De caserío agricola a vivienda rural: Evolución de la función agraria en la comarca de Donostia-San Sebastián, Departamento de Agricultura y Pesca. Gobierno Vasco, VitoriaGasteiz.

AlBizU, A. (1999): "Principales caractéristiques du secteur agro-alimentaire du Pays Basque, structures et outils de gestion pour una politique basée sur la qualité", La qualité au service du développement durabie, Euromontana, Vitoria-Gasteiz, pp. 51-53.

AirRUT, M. (1999): "Le rôle et l'utilisation des mesures communautaires pour un développement rural basé sur la qualité. Les choix d'une région autonome: exemple du Gouvernement Basque-espagnol", La qualité au service du développement durable, Euromontana, Vitoria-Gastez, pp. 101-104.

BERARD, L. (1996): "La construcción social de los productos de la tierra". Agricultura y Sociedad, $n^{\circ} 80$ 81, p. $31-56$.

Bérard, L.; Beucherie, O.; Fauvet, M.; Marchenay, P.; Monticelli, C. (2001): "Les facteurs historiques, culturels, économiques et environnementaux dans la délimitation des zones IGP ${ }^{2}$, Ingénieries, $n .^{\circ} 28$, pp. 49-63.

EMPRESA NACIONAL MERCASA (1999): Estudio para la remocielación del mercado de San Martín e implantación de nuevas actividades comerciales (Donostia-San Sebastián). Inédito. Ayuntamiento de San Sebastián.

ESPEITX BERNAT, E. (1996): "Los nuevos consumidores o las nuevas relaciones entre campo y ciudad a través de los productores de la tierra". Agricultura y Sociedad, n. ${ }^{\circ} 80-81$, pp. 83-116.

Galdos URRUTIA, R. (2003): Una estrategia para la sostenibilidad de las pequeñas explotaciones agrarias: la promoción de los productos agroalimentarios de calidad en el País Vasco (España). XI Annual Conference of the Comission of the International Geographical Union. Rio de Janeiro. En imprenta.

GATIK CONSULTORES (2002): Situación de la horticultura en el territorio de Vizcaya. Inédito.

GOBIERNO VASCO. Departamento de industria, Agricultura y Pesca (1998): Programa sub-sectorial de frutas y hortalizas. Inédito. Vitoria-Gasteiz.

Gómez, B.; ARmesto, X. A. (2002): "Turismo, gastronomia y territorio", Actas del XI Coloquio de Geografia Rural. Los espacios rurales entre el hoy y el mañana, Universidad de Cantabria, Santander, pp. $139-147$. 
HAGINA (1993 .... ): La revista Agricola para la Cornisa Cantábrica. Oiartzun (Guipúzcoa).

HERNANDEZ HERNANDEZ, M. (2000): “La agricultura de montaña en Alicante: situación actual y potencial futuro". Investigaciones Geográficas, n. ${ }^{\circ} 24$, p. 55-68.

HORTICULTURA (1995... ). Revista mensual. Ediciones de Horticultura. Reus (Tarragona).

MAULEÓN, J. R. (1998) Estrategias familiares y cambios productivos del caserio vasco, Gobierno Vasco, Vitoria-Gasteiz.

MINISTERIO DE AGRICULTURA, PESCA Y ALIMENTACIÓN (1995) Alimentos de España. Denominaciones de origen y de Calidad, Madrid.

RAMOS LEAL, F. (1991): "Estrategias de la distribución alimentaria: perspectivas para el sector horticola español». Revista de Estudios Agro-Sociales, n. ${ }^{\circ}$ 157, pp. 153-178.

Ruiz Urrestarazu, E. y Galdos UrRutia, R. (1987): "La horticultura intensiva en el Pais Vasco: los cultivos de invernadero". Anales de Geografía de la Universidad Complutense, VII (Homenaje al Profesor Manuel de Terán), pp. 325-333.

STUCKI, E. W. (1998): “Les démarches liées aux produits de qualité ancrés dan les terroir. Témoignage de Suisse", Développement dans les espaces à faible densité, Ingénieries. EAT, n. ${ }^{\circ}$ hors série, pp. 51-55. 